\title{
Designing an Ultra-Low-Power RTU for Use in NB-loT Water Applications
}

\author{
Ivan Ganchev ${ }^{1,2,3^{*}}$, Zhanlin $\mathrm{Ji}^{1,4}$, and Máirtín O'Droma ${ }^{1}$ \\ ${ }^{1}$ Telecommunications Research Centre (TRC), University of Limerick, Ireland \\ 2Department of Computer Systems, University of Plovdiv "Paisii Hilendarski", Plovdiv, Bulgaria \\ ${ }^{3}$ Institute of Mathematics and Informatics, Bulgarian Academy of Sciences, Sofia, Bulgaria \\ ${ }^{4}$ North China University of Science and Technology, Tangshan, China
}

\begin{abstract}
This paper presents the design and realization of a ultra-low-power and low-cost remote transfer unit (RTU), working as an outdoor gateway for collecting hydrographic data, such as rainfall, water flow rate, water quality, etc. Based on the NarrowBand Internet of Things (NB-IoT) standard, it facilitates the communication between the sensors and the information center (server). The unit consists of an Advanced RISC Machine (ARM) microcontroller unit (MCU), a NB-IoT module, and a power supply module. The RTU was experimentally tested and its use successfully demonstrated.
\end{abstract}

\section{Introduction}

The UN World Water Development Report 4 has declared that there will be 1.8 billion people faced with water scarcity by 2025 [1]. To save water, lots of countries have started building 'sponge cities', e.g. Tokyo (Japan), XiAn (China), Berlin (Germany), etc. The 'sponge city' concept aims to allow the absorption, infiltration, storing, purifying, draining, and managing rain water, storm water, ground water, etc. in urban environments, and to regulate the water cycle as much as possible to mimic the natural hydrological cycle [2]. Each 'sponge city' should have appropriate planning, management and control means in place in order to adapt and maintain its infrastructural systems to collect, store, and purify excess water. A very important role in this plays a real-time hydrologic information intelligence system, supporting the decision-making process of the city government.

In China, the State Council has set a progressive target for the 'sponge city' initiative to be achieved at the national level by 2030 [2]. In addition, the Hebei province's government has released a "Five-year implementation plan for comprehensive treatment of ground water over-exploitation (2018-2022)" in order to battle the over-pumping of ground water which caused ground subsidence. Accordingly, there will be 50,000+ monitoring points installed at the end of this period, each powered by a 65 -Ah nickel metal hydride battery with a 30-Watt solar panel, communicating with an information center via a General Packet Radio Service (GPRS) network, and costing around 16,000 Chinese Yuan $(\sim 200 €)$, Figure 1. Each monitoring point is able to collect hydrographic data, such as rainfall, water flow rate, depth of water, water quality, etc.
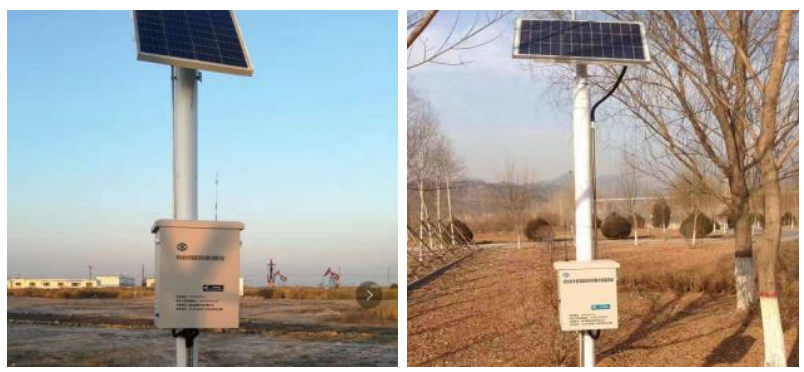

Fig. 1. Ground-water over-exploitation monitoring points.

With the rapid development of the NarrowBand Internet of Things (NB-IoT) technology [3], the GPRS will be replaced soon globally in all IoT application domains. IoT water applications are entering the micropower consumption era. Today a small 19-Ah battery can support the outdoor operation of a water monitoring station for more than 3 years.

Supplemented by big-data techniques and artificial intelligence algorithms, the grid monitoring and control of all water sources are becoming the primary goal for each (city) government. The Remote Transfer Unit (RTU) is the key module of each monitoring point, being part of such IoT system.

In [4], a GPRS-based data transfer unit is proposed as a communication module for use in an agricultural irrigation \& fertilization intelligent control system. [5] proposes a water quality automatic monitoring system, utilizing a GPRS-based unit. However, the power consumption of these units is at a $\mathrm{mA}$ level.

In this paper, a specially designed ultra-low-power and low-cost RTU is proposed for use in NB-IoT water applications. It includes a direct current to direct current (DC-DC) power supply module, two low dropout (LDO) regulator modules, a NB-IoT module, an isolating TIA-

\footnotetext{
* Corresponding author: ivan.ganchev@ul.ue
} 
485 module, an optical coupling isolation input module, and an Advanced RISC Machine (ARM) microcontroller unit (MCU). The MCU is a small system-on-a-chip (SoC) computer. A variety of MCUs are produced, e.g. the Advanced RISC Machine (ARM) core processors, Atmel AVR, Texas Instrument TI MSP, Intel 8051, etc. Among these, the 32-bit ARM processors (licensed by the ARM Holdings PLC) are the most widely used. These include Cortex M0, M1, M3, M4, and M7. The ultra-low-power STM32L151 - with a 64 Kbytes Flash, $32 \mathrm{MHz}$ CPU - costs only $1 €$, but provides portable, scalable, reliable, and efficient solution for embedded software design. These are the reasons behind selecting it as the MCU for the RTU described in this paper.

\section{Architectural Design}

Figure 2 shows a ground-water monitoring installation with a RTU installed in an Ingress Protection 68 (IP 68) box and hanged under the well lid. The module collects periodically (1 to 6 hours) a hydrologic data via a TIA485 serial port and sends it to an information center in the cloud.

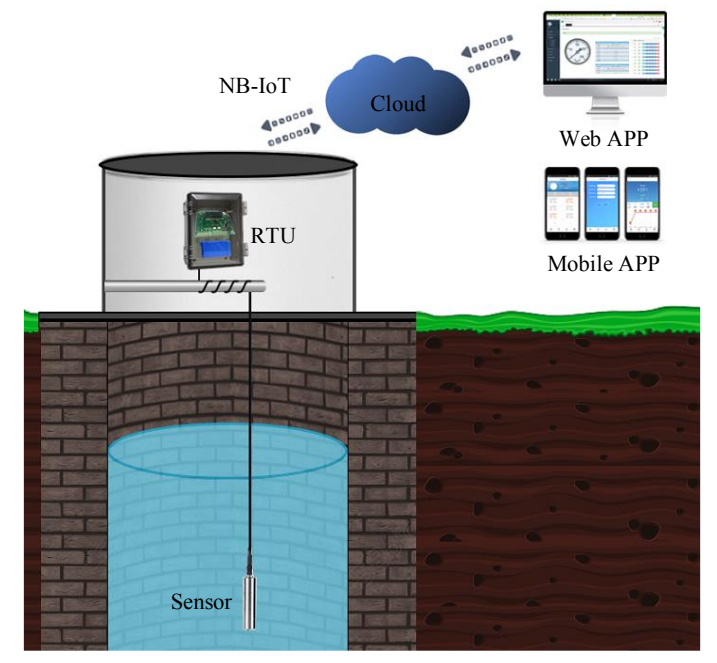

Fig. 2. A ground-water monitoring installation with an ultralow-power RTU.

In the RTU, the data is encapsulated into a Modbus RTU message, shown in Figure 3. The unit adds a Modbus application (MBAP) header and sends the resulting data unit to an information center (in the cloud) via TCP/IP protocols. After receiving a message from the center, it decapsulates the Modbus RTU message and processes the command, e.g., for RTU parameter updating, time synchronizing, water level gauge data reading, sensor parameters adjusting, etc.

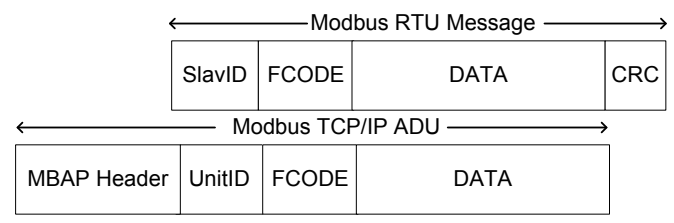

Fig. 3. The Modbus RTU message and the TCP/IP application data unit (ADU).
The hardware of the designed RTU includes three main modules:

- MCU Module: The STM32L151 32-bit MCU was selected for this module. It includes a 12-bit Analog-toDigital Converter (ADC), and two ultra-low-power comparators. In a stop mode, it only consumes $1.2 \mu \mathrm{A}$. The 64-pin MCU version with a Low-profile Quad Flat Package (LQFP) was chosen for use;

- NB-IoT Module: The Quectel BC28 NB-IoT was selected for this module due to its $1.77 \times 1.58 \mathrm{~cm}$ dimension, which meets the space requirement of the hardware design. Moreover, it only needs $500 \mathrm{~mA}$ current in a data burst mode and $3 \mu \mathrm{A}$ in a power safe mode with $3.3 \mathrm{~V}$ supply. The Universal Synchronous/Asynchronous Receiver/Transmitter (USART) serial port is utilized for communication with the MCU;

- Power Supply Module: The power supply supports $7-14.4 \mathrm{~V}$ input. Two LDO provide 3.3V VCC for the MCU and peripherals. A DC-DC unit provides $3.6 \mathrm{~V}$ VCC for the NB-IoT module.

\section{Implementation}

\subsection{MCU Module}

The STM32L151 schematic design is shown on Figure 4. The MCU can be waked up from a low-power sleep mode when receiving a rising edge on pin PA0 (I2C calendar) or pin PB5 (digital input). Then the MCU calculates the power voltage from pins PA1 and PA3. If the voltage can power up the sensors, the MCU enables the power MOSFET NTS4101PT1G via pin PB15 and collects data via the TIA-485 serial port. After generating a data frame, the latter is sent to the NB-IoT module via the USART port. The MCU saves the data to EEPROM via the SPI-bus, i.e., pins PA5-PA7.

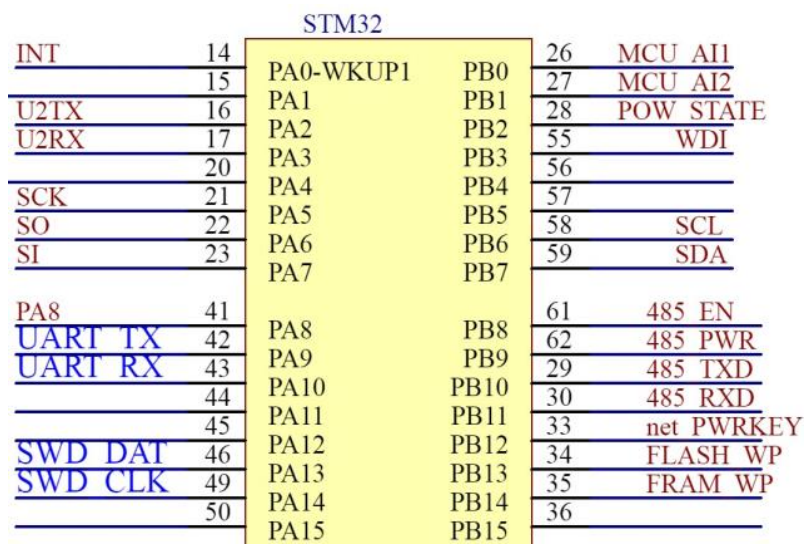

Fig. 4. The MCU schematic design.

The I2C (pins PB6 \& PB7) connects the PCF8563 unit (which provides a CMOS real-time clock and calendar) with the MB85RC64 unit (which is a ferroelectric random access memory chip for saving the important parameters of the RTU), Figure 5. The essential feature of PCF8563 and MB85RC64 is the low- 
power consumption as the operating supply current is at an $\mu \mathrm{A}$ level.

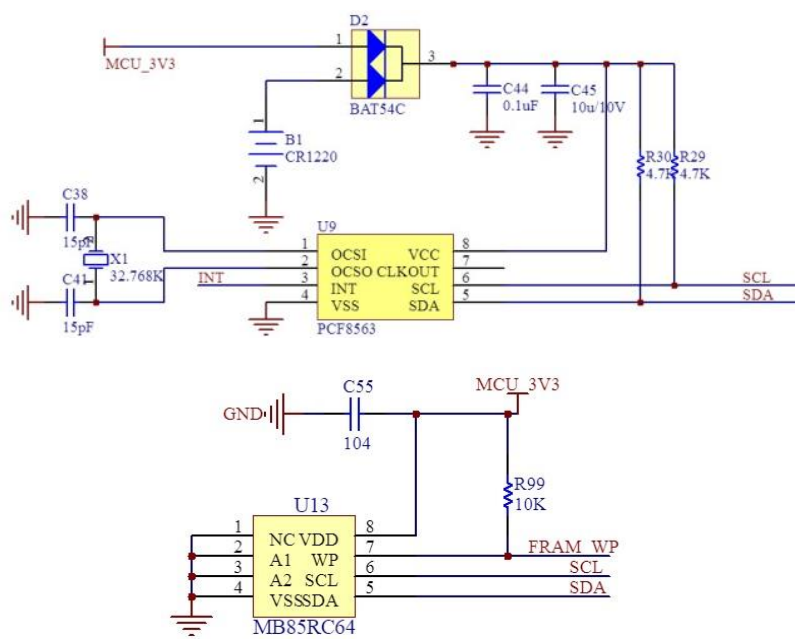

Fig. 5. The I2C schematic design.

The TIA-485 serial port's schematic design is shown in Figure 6. The power is controlled via pin PB9 by NTR4101.
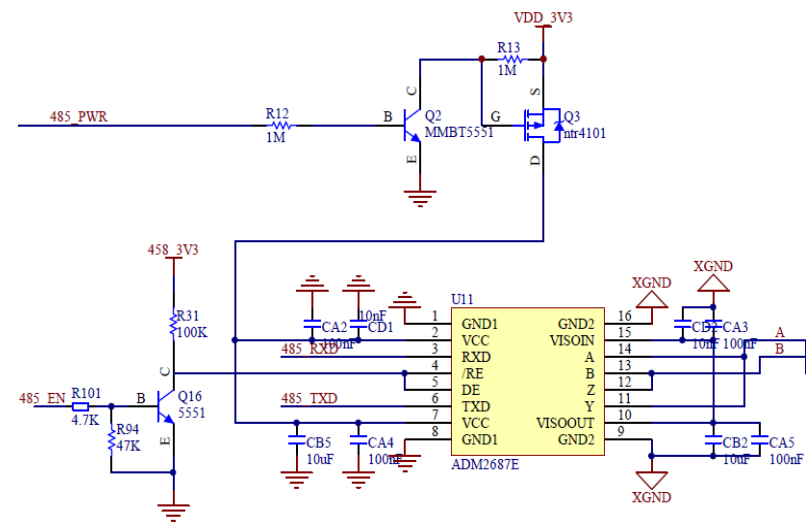

Fig. 6. The TIA-485 serial port's schematic design.

\subsection{NB-IoT Module}

The NB-IoT module is used for communication with the external world, facilitated by a $\mathrm{BC} 28$ unit that includes RX/TX ports for connection with PA2 and PA3, a GSM ANT port for connection with the antenna, and a SIM-ports slot part for the SIM card. The power supply part includes four bypass capacitors - a $100-\mu \mathrm{F}$ tantalum capacitor and three ceramics capacitors (Figure 7).

\subsection{Power Supply Module}

Beside 3.3V LDOs, the NB-IoT module needs a $3.6 \mathrm{~V}$ DC support. Figure 8 shows the schematic design of the power supply module.

\section{Results}

The 4-layer RTU hardware PCB design is shown on Figure 9a. Its size is $4 \times 10 \mathrm{~cm}$, and thus could be easily inserted (along with the batteries) into a steel pipe with a 48-mm diameter, as shown on Figure 9b. Four 3.6V dry batteries are used to achieve a 14.4-V (19-Ah) value.

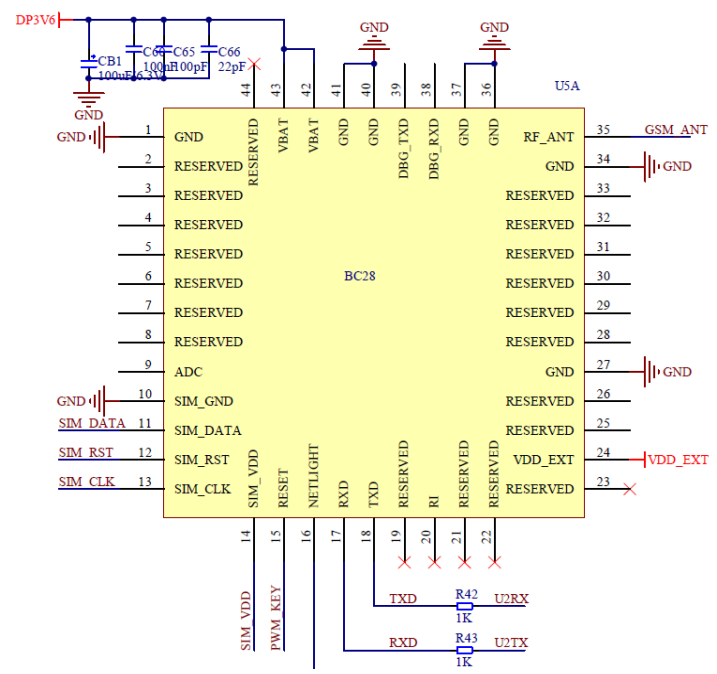

Fig. 7. The $\mathrm{BC} 28$ schematic design.

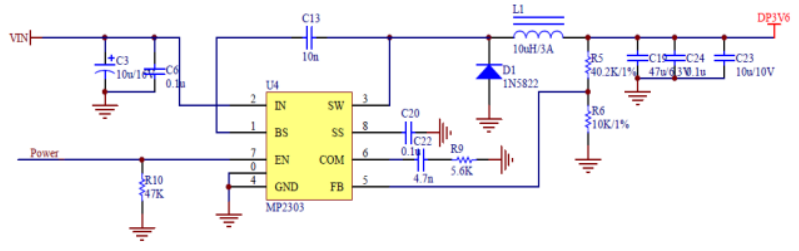

Fig. 8. The power supply module's schematic design.

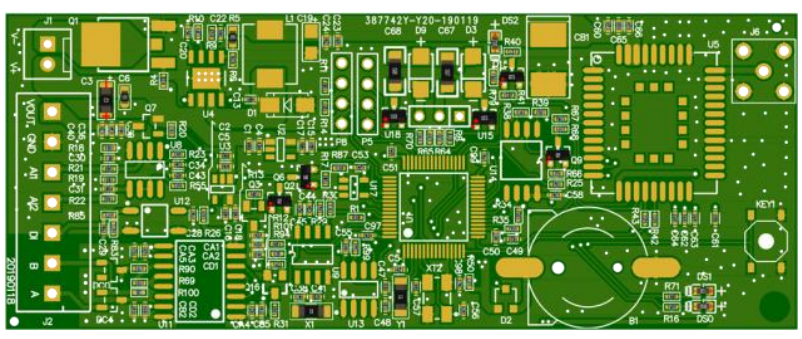

(a)

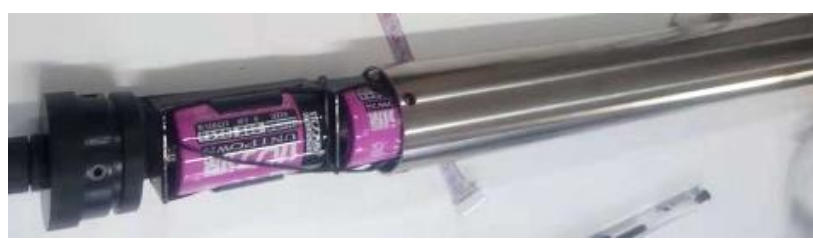

(b)

Fig. 9. The RTU hardware: (a) the PCB design; (b) the 48-mm steel pipe installation.

The cost of the designed RTU hardware is around 160 Chinese Yuans ( 20€). It includes 65 different electronic components. Figure 10 shows the RTU running in an ultra-low-power mode (the quiescent current is $7.5 \mu \mathrm{A}$ ).

A Java-based software platform has been developed for the information center (server) in order to do experiments with the proposed RTU. A corresponding web application and an Android-based mobile application (Figure 11) have been developed for hydrologic monitoring. 


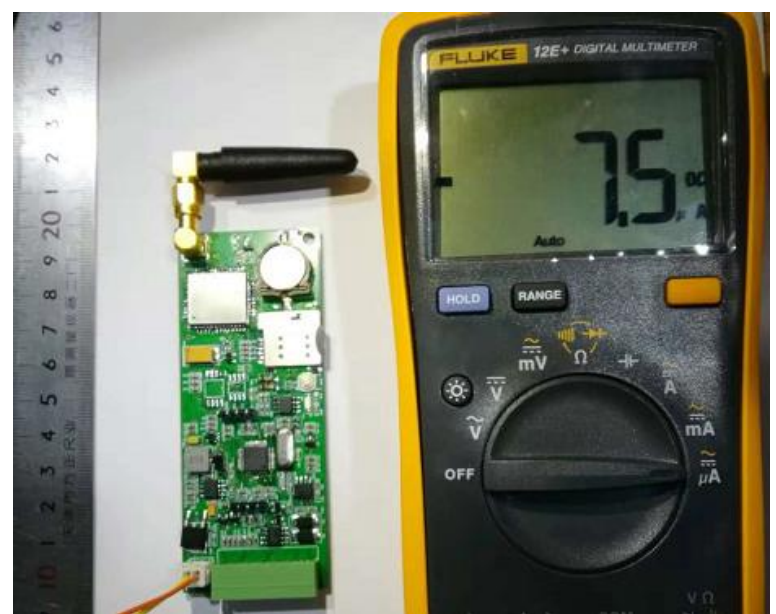

Fig. 10. The RTU running in an ultra-low-power mode (with a quiescent current of $7.5 \mathrm{~A}$ ).

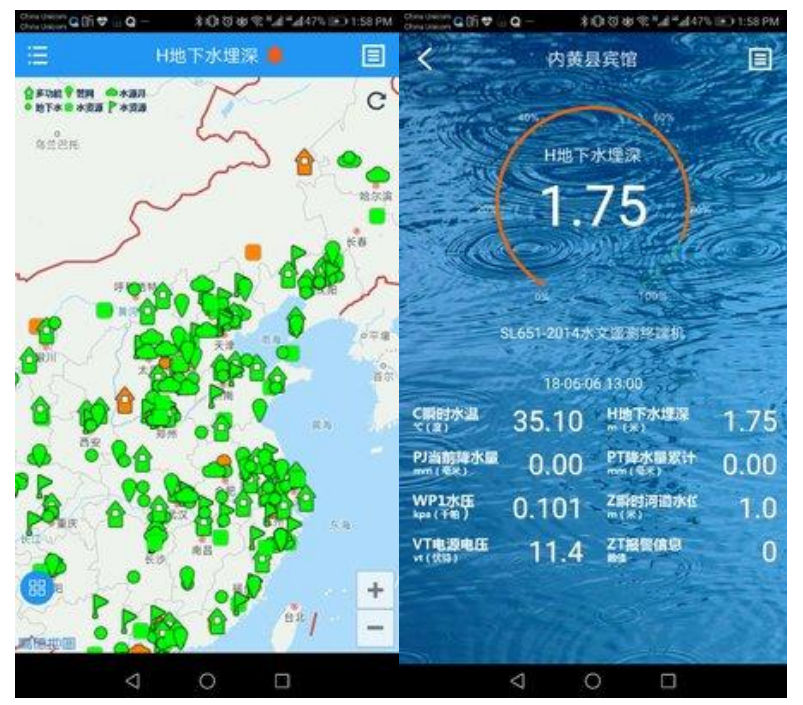

Fig. 11. The developed Android-based mobile application.

\section{Conclusion}

An ultra-low-power and low-cost remote transfer unit (RTU) for use in IoT water applications has been presented in this paper. The unit utilizes the NarrowBand Internet of Things (NB-IoT) technology for supporting the communication between hydrologic sensors and an information center. The hardware module consists of a STM32L151 microcontroller unit (MCU), a power supply module, and a BC28-based NB-IoT module. The RTU was experimentally tested and its use successfully demonstrated.
This work has been accomplished with the financial support of the MES by the Grant No. DO1-221/03.12.2018 for NCDSC part of the Bulgarian National Roadmap on RIs, the NSF of the Hebei Province, China, Grant No. F2018209358, and the S\&T Major Project of the Science and Technology Ministry of China, Grant No. 2017YFE0135700.

\section{References}

1. WWAP, The United Nations world water development report 4: Managing water under uncertainty and risk, ed: UNESCO Paris, (2012)

2. C. Zevenbergen, D. Fu, A. Pathirana, Transitioning to Sponge Cities: Challenges and Opportunities to Address Urban Water Problems in China, Water, 10:1230 (2018)

3. Y.-P. E. Wang, X. Lin, A. Adhikary, A. Grovlen, Y. Sui, Y. Blankenship, et al., A primer on 3GPP narrowband Internet of Things, IEEE Commun.Mag, 55:117-123 (2017)

4. Y. T. Wang, H. X. Zhang, J. C. Li, Y. Y. Wu, Application and Research of Agricultural Irrigation Fertilization Intelligent Control System Based on GPRS DTU, AMM, 441:783-787 (2014)

5. W. Dehua, L. Pan, L. Bo, and G. Zeng, Water quality automatic monitoring system based on GPRS data communications, Procedia Eng, 28: 840843 (2012) 